\title{
THE EFFECT OF CRYSTALLINE ORIENTATION ON VIBRATION SENSITIVITY OF SILICON MICRO-RESONATORS
}

\author{
B.Khazaeili, and R. Abdolvand \\ University of Central Florida, Orlando, Florida, USA
}

\begin{abstract}
In this work the effect of crystalline orientation on the acceleration sensitivity of Silicon-based MEMS oscillators is experimentally studied for the first time. The thin-film piezoelectric-on-Silicon (TPoS) platform is utilized to implement the oscillators as it enables resonators with low-motional resistance and high $Q$. A single lateral-extensional-mode resonator design is fabricated in $\langle 100\rangle$ and $\langle 110\rangle$ orientations on a $<100\rangle$ Silicon wafer. The resonators are then used to assemble two oscillators operating at $\sim 25 \mathrm{MHz}$ and $\sim 27 \mathrm{MHz}$ respectively. The average acceleration sensitivity of the oscillator containing the $<110>$ resonator is measured to be $\sim 4 \times 10^{-10}$ at vibration frequencies up to $2700 \mathrm{~Hz}$; an astonishing two orders of magnitude lower than that of the oscillator utilizing the $<100>$ resonator. The acceleration sensitivity in these Silicon-based resonators is believed to stem from nonlinear elastic properties of Silicon, which is dependent on crystalline orientation as well as doping type/concentration. The Silicon substrate used in this work is Phosphorous-doped at $\sim 5 \mathrm{e} 19$ $\mathrm{cm}^{-3}$ concentration.
\end{abstract}

\section{INTRODUCTION}

High-performance ultra-stable oscillators are of great interest in wireless communication applications. Frequency stability of the oscillators that utilize mechanical resonators can be affected by the environment vibrations especially in harsh mobile systems like radars and cell-phone towers. Therefore, acceleration sensitivity is an important characteristic of the resonator chosen for such applications.

The acceleration sensitivity of quartz resonators and their dependency on the crystalline orientation have been extensively studied [1]. With the emergence of micromachined Silicon resonators as a viable alternative to quartz in applications traditionally dominated by quartz, a thorough study of vibration sensitivity in Silicon resonators is in order. Some attention have been recently directed toward studying the acceleration sensitivity of the Silicon-based MEMS resonators [2-6] but the role crystalline orientation could play in the outcome has yet to be considered.

In general, the level of acceleration sensitivity in a resonator depends on several factors such as the material chosen for the resonant body, dimensions of the resonator, resonance mode, and anchor arrangement [7]. Most studies exploring the effect in microresonators have focused on strategies to reduce the vibration sensitivity in capacitively-transduced resonators. The dominant source of acceleration sensitivity in such resonators appear to be the variation of electrostatic stiffness (i.e. gap size) as a result of vibration. The reduction of sensitivity have been explored through strategically located anchors [2-5] and increasing the capacitive gap size [6].

Although capacitive resonators offer excellent quality factor, thin-film piezoelectric-on-Silicon (TPoS) resonators have been shown to exhibit significantly lower motional resistance and nonlinearity without excessively sacrificing the $Q$ [8]. Therefore, TPoS resonators are believed to offer competitive advantages compared to capacitive resonators for oscillator applications. In TPoS resonators and any class of MEMS resonators that don't operate based on capacitive transduction the acceleration sensitivity is yet to be carefully characterized and understood.

In this work, the effect of the Silicon crystalline orientation on the resonator vibration sensitivity is investigated. For this purpose TPOS resonators are fabricated in the two commonly accessible Silicon crystalline orientations: $\langle 100\rangle$ and $<110\rangle$. The measured results of this work suggest that the acceleration sensitivity of bulk mode resonators oriented in $<110>$ direction is about two orders of magnitude lower than that of resonators oriented in the $\langle 100\rangle$ direction. It should be noted that the acceleration sensitivity in such resonators is believed to be a function of the nonlinear elastic properties of Silicon. As shown by other work presented in the past including one published by our group [9], nonlinearity in Silicon is a sensitive function of doping type/concentration. Consequently the results of this work cannot be generalized for all Silicon resonators, and rather similar studies need to be performed for a specific doping type/concertation. The Silicon substrate used in this work is doped with Phosphorous at $\sim 5 \mathrm{e} 19 \mathrm{~cm}^{-3}$ concertation in order to improve the temperature stability as shown by our group in earlier studies [10].

The paper is organized as follows. First, the acceleration sensitivity of the resonator is mathematically described. Then, the resonators used in this study are characterized using a network analyzer before the experimental set up for measuring the resonators vibration sensitivity is presented. Finally, the measured acceleration sensitivity results are presented and discussed.

\section{ACCELERATION SENSITIVITY}

The resonance frequency of a resonator can change due to the strain perturbation induced by external acceleration. The value of this shift depends on the vibration magnitude, its direction and acceleration sensitivity of the resonator. Thus, the resonance frequency during vibration is given by [1]

$$
f(\vec{a})=f_{0}(1+\vec{\Gamma} \cdot \vec{a})
$$

where $f_{0}$ is the natural resonance frequency of the resonator, $\Gamma$ is the acceleration sensitivity vector and $\mathrm{a}$ is the applied acceleration. Based on (1), $\Gamma$ can be found by

$$
\vec{\Gamma}=\Delta f / f_{0} \vec{a}
$$

where $\Delta \mathrm{f}$ is the resonance frequency shift, which is usually a small value for resonators with low vibration sensitivity complicating the characterization efforts. One reasonable approach for measuring $\Gamma$ is to employ the resonator in an oscillator circuit and monitor the output frequency spectrum while an alternating external acceleration is applied to the oscillator. Let's assume the applied acceleration is:

$$
\vec{a}(t)=\vec{A} \cos \left(2 \pi f_{v} t\right)
$$

where $A$ is the maximum magnitude of the acceleration and $f_{v}$ is the vibration frequency. In this case the resonance frequency of the resonator should vary as follows:

$$
f(\vec{a})=f_{0}\left(1+(\vec{\Gamma} \cdot \vec{A}) \cos \left(2 \pi f_{v}\right)\right)
$$


On the other hand, consider the output signal of an oscillator as:

$V(t)=V_{0} \cos (\varphi(t))$

Where $\varphi(t)$ is the phase of the circuit and is obtained by

$\varphi(t)=2 \pi \int_{t_{0}}^{t} f\left(t^{\prime}\right) d t^{\prime}$

By substituting (4) in (6), the oscillator phase can be obtained as

$\varphi(t)=2 \pi f_{0} t+\left(\Delta f / f_{v}\right) \sin \left(2 \pi f_{v} t\right)$

Thus, the oscillator output signal will be

$V(t)=V_{0} \cos \left(2 \pi f_{0} t+\left(\Delta f / f_{v}\right) \sin \left(2 \pi f_{v} t\right)\right)$

Equation (8) indicates a frequency modulated signal at the oscillator output. By using the Bessel series to expand (8), The voltage can be expressed as

$$
\begin{aligned}
& V(t)=V_{0}\left[J_{0}(\beta) \cos \left(2 \pi f_{0} t\right)+J_{1}(\beta) \cos \left(2 \pi\left(f_{0} \pm f_{v}\right) t\right)\right. \\
& \left.\left.+J_{2}(\beta) \cos \left(2 \pi\left(f_{0} \pm 2 f_{v}\right) t\right)\right)+\ldots\right]
\end{aligned}
$$

In which $\beta=\Delta f / f_{v}=(\Gamma \cdot A) f_{0} / f_{v}$ is the modulation index. For $\beta<0.1$, $J_{0}(\beta)=1, J_{1}(\beta)=\beta / 2$, and for $\mathrm{n}>1 \mathrm{~J}_{\mathrm{n}}(\beta)=0$. Thus, in the oscillator output spectrum, there will be two sidebands at $\mathrm{f}_{0} \pm \mathrm{f}_{\mathrm{V}}$ besides the carrier signal at $f_{0}$. The ratio of the sidebands power to the carrier power can be derived by

$$
L_{v}=20 \log \left(J_{1}(\beta) / J_{0}(\beta)\right)=20 \log \left((\vec{\Gamma} \cdot \vec{A}) f_{0} /\left(2 f_{v}\right)\right)
$$

By rewriting (9), the acceleration sensitivity is obtained by

$$
\Gamma=\left(2 f_{v} / A f_{0}\right) 10^{L_{v} / 20}
$$

The acceleration sensitivity in oscillators that contain piezoelectric-based resonators is believed to stem from: 1) deformation of the resonator due to external forces (geometrical effects) 2) the nonlinear behavior of the material elastic properties with the latter being usually the dominant factor [7]. The external acceleration applied to the resonator acts on the resonator mass and generates a force that causes stress/strain in the device, which can either change the resonator geometry and/or the material stiffness if the elastic behavior of the material is nonlinear. As is shown by complicated theoretical expressions in [7] for bulk acoustic wave (BAW) micro-resonators the acceleration sensitivity is proportional to a complex sum of linear and nonlinear elastic stiffness coefficients. In addition, $\Gamma$ is inversely proportional to the linear stiffness coefficient of the material. Hence, a material with large linear elastic stiffness and low nonlinear elastic stiffness coefficients, can exhibit low sensitivity to external vibration.

Based on this concept, since the elastic behavior of single crystal Silicon is orientation-dependent, the acceleration sensitivity of resonators oriented to different crystalline planes is expected to be orientation-dependent as well.

\section{DEVICES CHARACTERIZAION}

To explore the effect of the crystalline orientation on acceleration sensitivity, two similar devices in terms of dimensions and design have been fabricated in two different crystalline orientations: $<110>$ and $<100>$. The resonators are two-port devices with an interdigitated top metal pattern that could excite both the fundamental and the third harmonic lateral-extensional modes. The resonators are fabricated on an $\sim 8 \mu \mathrm{m}$ Phosphorusdoped (doping concertation $\sim 5 \times 10^{19} \mathrm{~cm}^{-3}$ ) silicon-on-insulator (SOI) substrate with an stack of Mo/AlN/Mo $(100 \mathrm{~nm} / 1000 \mathrm{~nm} / 100 \mathrm{~nm})$ deposited for the piezoelectric transduction mechanism [10]. Note that the substrate in this study is a $<100>$ SOI wafer, so the resonators aligned in parallel with the wafer flat are oriented in the $<110>$ direction, while the ones at a $45^{\circ}$ angle with respect to the flat are oriented to the $<100>$ plane. The relative layout of the resonators on the wafer are shown in Figure 1. In addition, the fundamental lateral-extensional mode shape of the $<100>$ TPOS resonator simulated by COMSOL and the direction of applied acceleration $\left(\mathrm{a}_{\mathrm{z}}\right)$ are shown in the inset diagram.

The frequency response, measured by a Rohde \& Schwarz ZNB 8 Network Analyzer, and scanning electron micrograph (SEM) of the two resonators used in this study are shown in Fig. 2. The response at fundamental resonance mode of the resonators is presented since that will be the mode utilized in the oscillator. As seen, the resonance frequency of the device in $\langle 110\rangle$ direction is higher than the one in $<100>$ direction because of the larger Silicon Young's modulus in $<110>$ orientation. Quality factor and motional resistance of $<100>$ and $<110>$ resonators are $4.639 \mathrm{~K}$, $322.18 \Omega$ and $9.975 \mathrm{~K}, 236.97 \Omega$ respectively.

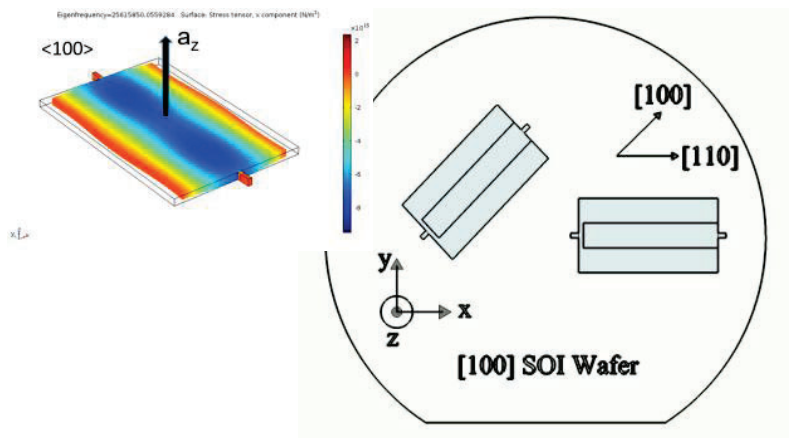

Figure 1: The layout of $\langle 100\rangle$ and $\langle 110\rangle$ resonators relative to the wafer and the simulated mode shape of the $<100>$ resonator.
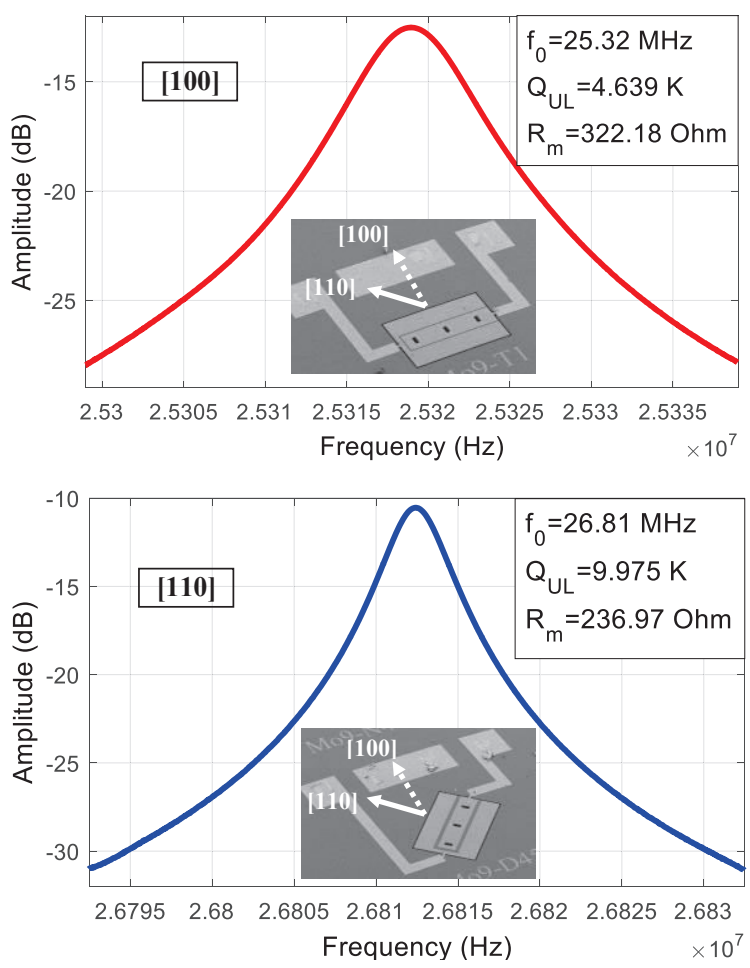

Figure 2: Frequency response and SEM of fabricated resonators in $<100>$ and $<110>$ orientation. 


\section{EXPERIMENTAL RESULTS}

In order to measure the acceleration sensitivity of the resonators using equation (11), they should operate in an oscillator circuit. Therefore, two identical printed circuit boards (PCB) have been assembled. The oscillator circuit is based on a CF5027 IC module commonly used in commercial quartz oscillators. The CF5027 IC is mounted on a ceramic leadless chip carrier (LCC) package and the resonator has been connected to it in a one-port configuration to keep the phase of the oscillator loop equal to $0^{\circ}$. Care has been taken to reduce the number of electronic components, the size of the board, and the length of the wirebonds in order to minimize the parasitic effects.

A Rohde \& Schwarz FSUP Signal Source Analyzer is used to capture the oscillators phase noise plot. Both oscillators exhibit excellent phase noise performance with the $\langle 110\rangle$ oscillator having an slightly better close-to-carrier performance (Fig. 3) due to the higher Q (-125 vs. - $120 \mathrm{dBc} / \mathrm{Hz} @ 1 \mathrm{kHz}$ offset).

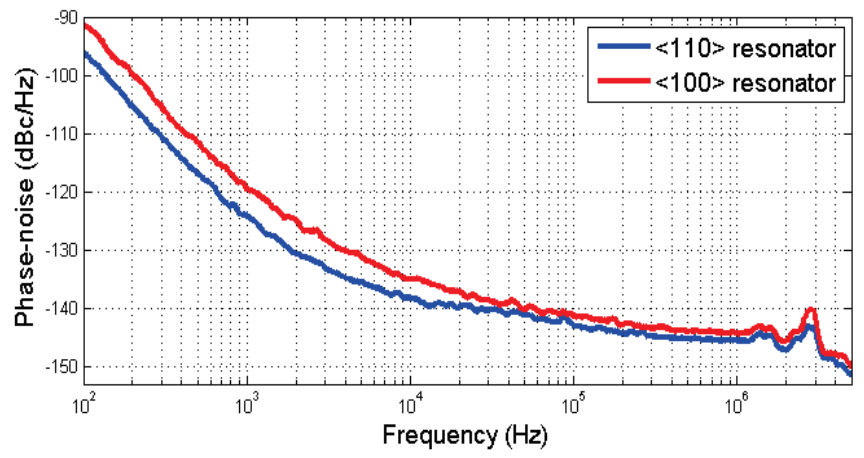

Figure 3. Oscillator phase noise for both resonators.

The vibration measurement setup is a closed-loop system including a magnetic motor, a pa-138 amplifier, a VR9500 controller and a DYTRAN 3055B1T accelerometer from Vibration Research Corporation. The accelerometer is mounted on the oscillator board to measure the actual acceleration that is fed back to the controller. Then the controller compares the measured acceleration of the board with the desired value of acceleration, which has been set by the operator, and provides a proper signal for the amplifier. Based on this signal the amplifier generates the required gain for the magnetic motor to reach the desired displacement and acceleration. The PCB of the oscillator and the vibration system used in this work are shown in Figures 4 and 5 respectively.

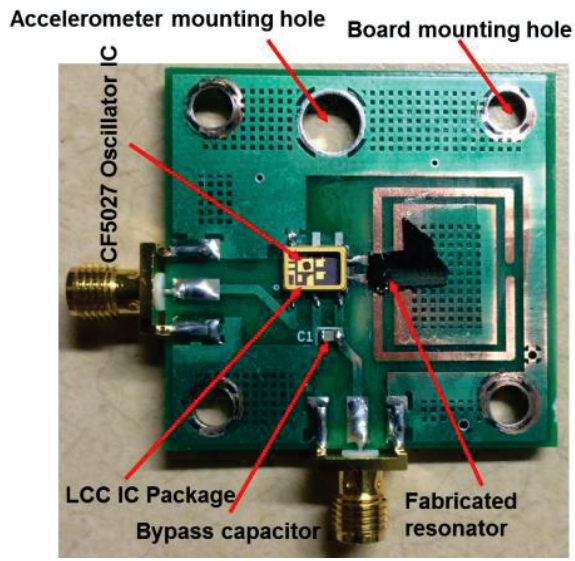

Figure 4. The oscillator board

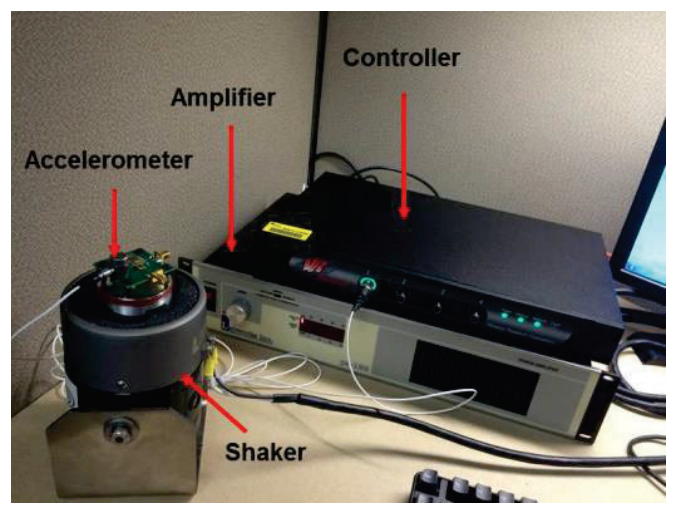

Figure 5. The vibration sensitivity measurement setup.

Using this setup, a $6 \mathrm{~g} 1.5 \mathrm{kHz}$ sine-wave vibration in $\mathrm{z}$ direction, normal to the Silicon surface (see inset diagram in Fig. 1), has been applied to the resonators. The frequency spectrum of the two oscillator output signals, captured by the signal source analyzer is demonstrated in Fig. 6. As seen, the same acceleration has generated much smaller side-bands in the spectrum of the $\langle 110\rangle$ device compared with the $<100>$ resonator. Using (11), acceleration sensitivity in z-direction is calculated as $\Gamma \mathrm{z},<100>=3.3 * 10^{-8}$ and $\Gamma \mathrm{z},<110>=5 * 10^{-10}$ in $\langle 100>$ and $<110>$ directions, respectively.
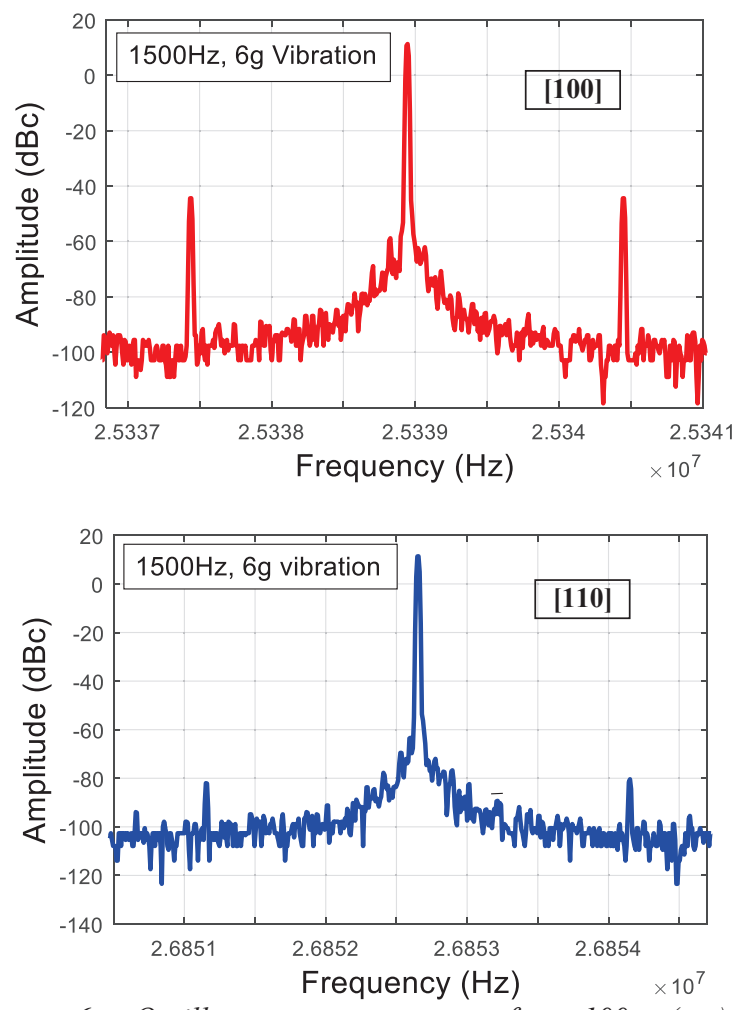

Figure 6. Oscillator output spectrum for $<100>$ (top) and $<110>$ (bottom) resonators under a $6 \mathrm{~g} 1.5 \mathrm{kHz}$ vibration.

The acceleration magnitude and frequency is then kept constant $(7 \mathrm{~g}$ and $2 \mathrm{kHz})$ to obtain the frequency and amplitude dependency of the acceleration sensitivity (Fig. 7 and Fig. 8, respectively). As seen, for different vibration magnitudes and 
frequencies, the resonator in $<110>$ direction continues to exhibit two orders of magnitude less sensitivity to external vibration in comparison with $<100>$ resonator.

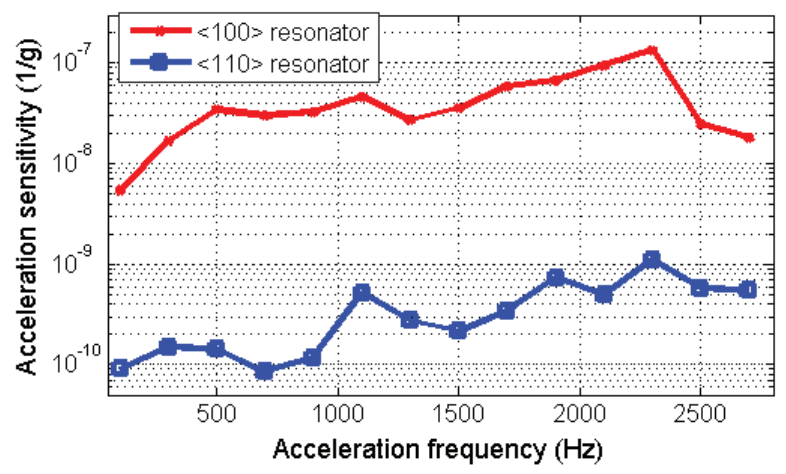

Figure 7. Acceleration sensitivity versus vibration frequency

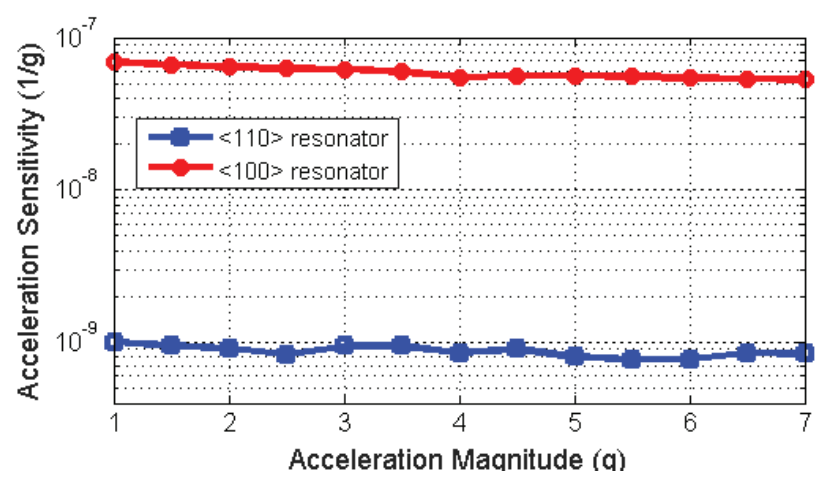

Figure 8. Acceleration sensitivity versus acceleration magnitude

\section{RESULTS DISCUSSION}

Based on the observations of this work, the acceleration sensitivity of a resonator made of highly-doped n-type silicon substrate is much less if it is oriented to the $<110>$ crystalline plane compared to one oriented to the $\langle 100\rangle$ plane. Considering that the acceleration sensitivity is believed to be dominated by the nonlinear elastic properties of the resonant body as described earlier in this paper, the results of this work are in agreement with the previous reports on nonlinearity of bulk-mode silicon resonators aligned to different crystalline orientations [11]. As reported in [11], the nonlinear behavior of bulk-mode resonators depends on the doping level, dopant type, mode-shape and crystalline orientation. Moreover, a length extensional mode resonator with n-type Phosphorous doped silicon substrate, devices aligned to $<110>$ plane exhibit lower nonlinearity compared with the devices aligned to $\langle 100>$ plane. Hence the reported results of this work are qualitatively anticipated.

\section{CONCLUSION}

In this paper, the acceleration sensitivity of silicon-based microresonators aligned to different Silicon crystalline orientation is investigated. Two identical resonators are fabricated on a highlydoped n-type silicon wafer aligned to $\langle 100\rangle$ and $\langle 110\rangle$ crystalline planes. These resonators are employed in two identical oscillator circuits in order to measure their acceleration sensitivity. By applying a sine-wave vibration and measuring the oscillator output power at the resonance frequency and sidebands at the vibration frequency offset, the vibration sensitivity of the resonators were characterized. The obtained results indicate that the resonator aligned to $<100>$ direction is more sensitive to external vibration than the one aligned to $<110>$ direction. Since the dominant source of acceleration sensitivity in piezoelectric resonators is the nonlinear elastic properties of material, our observations confirm that nonlinear stiffness coefficients in $<100>$ orientation are larger than that of $\langle 110\rangle$ orientation in highly n-type-doped Silicon. Note that this result may be different for a different doping type/concentration since Silicon nonlinearity depends on these parameters.

\section{ACKNOWLEGEMENT}

Authors wish to thank Jonathan Gonzalez for his contribution in the fabrication of the resonators. This work was partially funded by NSF grant ECCS/1440163.

\section{REFERENCES}

[1] R.L. Filler, "The acceleration sensitivity of quartz crystal oscillators: a review," IEEE Transactions on Ultrasonics, Ferroelectrics, and Frequency Control, vol.35, no.3, pp.297305, 1988.

[2] T.L. Naing, T.O. Rocheleau, R. Zeying, E. Alon, C.T Nguyen, "Vibration-insensitive 61-MHz micromechanical disk reference oscillator," IEEE International Frequency Control Symposium (IFCS), pp.1-6, 2012.

[3] B. Kim, R.H. Olsson, K. Smart, K.E. Wojciechowski, "MEMS resonators with extremely low vibration and shock sensitivity," IEEE Sensors, pp.606-609, 2011.

[4] B. Kim, M. Akgul, Y. Lin, W. Li, Z. Ren, and C.T.-c. Nguyen, "Acceleration sensitivity of small-gap capacitive micromechanical resonator oscillators," IEEE International Frequency Control Symposium (IFCS), pp.273-276, 2010.

[5] K.E. Wojciechowski, R.H. Olsson, M.S. Baker, J.W. Wittwer, "Low Vibration Sensitivity MEMS Resonators," IEEE International Frequency Control Symposium Joint with the 21st European Frequency and Time Forum, pp.1220-1224, 2007.

[6] M. Agarwal, K.K. Park, M. Hopcroft, S. Chandorkar, R.N. Candler, B. Kim, R. Melamud, R., G. Yama, B. Murmann, T.W. Kenny, "Effects of Mechanical Vibrations and Bias Voltage Noise on Phase Noise of MEMS Resonator Based Oscillators," IEEE International Conference on Micro Electro Mechanical Systems,(MEMS), pp.154-157, 2006.

[7] J.A. Kosinski, "The fundamental nature of acceleration sensitivity," IEEE International Frequency Control Symposium, pp.439-448, 1996.

[8] B. P. Harrington, M. Shahmohammadi, and R. Abdolvand, "Toward ultimate performance in GHz MEMS resonators: loe impedance and high Q," IEEE International Conference on Micro Electro Mechanical Systems (MEMS), pp.707-710, 2010.

[9] M. Shahmohammadi, H. Fatemi, and R. Abdolvand, "Nonlinearity reduction in silicon resonators by doping and re-orientation," IEEE International Conference on Micro Electro Mechanical Systems (MEMS), pp.793-796, 2013.

[10] M. Shahmohammadi, B. P. Harrington, and R. Abdolvand, "Turnover temperature point in extensional-mode highly doped silicon microresonators," IEEE Transaction on Electron Devices, 60, 3, pp.1213-1220, 2013.

[11] Y. Yang, E. J. Ng, V. A. Hong, C. H. Ahn, Y. Chen, E. Ahadi, M. Dykman, and T. W. Kenny, "Measurement of the nonlinear elasticity of doped bulk-mode MEMS resonators," Solid-State Sensors, Actuators, and Microsystems Workshop, Hilton Head 2014, pp.285-288, 2014.

\section{CONTACT}

*beheshte.khazaeli@gmail.com 